\title{
Usos y percepciones de la fauna silvestre por pobladores de dos barrios aledaños a la Reserva Natural Otamendi, Campana, Argentina
}

\author{
Diana P. CRuz ${ }^{\bowtie}$ \& Paula Courtalon ${ }^{\bowtie}$ \\ Grupo de Investigación en Ecología de Humedales (GIEH). Departamento de Ecología Genética y Evolución Facultad de \\ Ciencias Exactas y Naturales. Universidad de Buenos Aires. Ciudad Universtaria. Pabellón 2. 4to piso, lab. 57. Buenos Aires, \\ Argentina.
}

\begin{abstract}
Resumen. La Reserva Natural Otamendi (RNO), ubicada en el partido de Campana, Provincia de Buenos Aires, posee ambientes propios de la Región Pampeana y del Delta del Paraná. La matriz del paisaje está formada por agroecosistemas, y la RNO preserva, por ejemplo, parches de pastizal pampeano, parches de bosque de tala (Celtis tala) y parches de bosque ribereño. En la comunidad aledaña a la reserva se evaluaron las referencias al uso de fauna silvestre y las percepciones de dicha fauna. Se llevaron a cabo entrevistas a pobladores locales, contactados a través de la Administración de Parques Nacionales. Además, para ampliar la muestra se utilizó la técnica "bola de nieve". Los resultados mostraron tres usos principales de la fauna silvestre: para alimento, para comercialización o como mascotas. Los mamíferos resultaron ser los animales más importantes para los pobladores que cazan fauna silvestre. Las especies más referenciadas y, por lo tanto, de mayor valor de uso fueron el coipo (Myocastor coypus), el carpincho (Hydrochaeris hydrochaeris) y el ciervo de los pantanos (Blastocerus dichotomus). Se constató la práctica de la caza en la zona aledaña a la RNO y se identificaron los conflictos que esta práctica genera entre los pobladores y las autoridades de la zona. A partir de este trabajo, se sugiere ampliar los estudios acerca de los usos de fauna en el área. Asimismo, se estima importante que en los espacios comunes de reunión de los pobladores locales se refuerce la divulgación del estado de conservación de las especies con valor de uso en la región, con el fin de establecer y evaluar bases sustentables para su conservación.
\end{abstract}

[Palabras clave: entorno rural, área protegida, fauna silvestre, comunidad]

\begin{abstract}
Aвstract. Wildlife uses and perceptions by residents from two surrounding neighborhoods of the Otamendi Natural Reserve in Campana, Argentina. The Otamendi Natural Reserve (ONR), located in Campana, Buenos Aires, has environments of the Pampa Region and the Paraná Delta. The landscape matrix in this area is composed by agroecosystems, the ONR for example, and preserves patches of Pampean pastureland, tala (Celtis tala) forest and riparian forest. In the community nearby the reserve, evaluation was made through the references made in the use of wildlife and people's perception of the mentioned fauna. Interviews were made to locals by the National Parks Administration and, in order to get a wider sample, the "snowball" technique was used. The results showed three main uses: as food, trade or as pets. For the people who hunt wildlife, mammals seem to be the most important animals. Three species were the most referenced, and so they represent a greater use value: the coipus (Myocastor coypus), the capybara (Hydrochaeris hydrochaeris) and the marsh deer (Blastocerus dichotomus). Hunting is confirmed in the surroundings of the ONR as well as conflicts between the authorities and locals of this activity. We suggest to extend the studies about wildlife use in this area and also, it is very important, among locals using people's communal areas, to strengthen the dissemination of the conservation status of species with high use value in the region, all this to establish and to evaluate sustainable basis for their conservation.
\end{abstract}

[Key words: rural environment, protected area, wildlife, people]

\section{INTRODUCCIÓN}

En los últimos años, en Latinoamérica surgió un interés por investigar cómo las diferentes culturas interactúan con su entorno (Racero et al. 2008; Navarro 2005). Una de las razones es porque los cambios naturales que se presentan en la población humana, tales como el incremento del acceso a la tecnología o la migración del campo a las ciudades, hacen que estas interacciones sean dinámicas en el tiempo. Específicamente, la interacción del hombre con la fauna ha sido objeto de estudio

Editor asociado: Pedro Blendinger

$\triangle$ dianapatriciacruz@gmail.com;

pcourtalon@gmail.com en varios lugares; por ejemplo, se plantea que en comunidades tradicionales es usual que prime la interacción a través del uso destinado al autoconsumo y distintas categorías de uso, como son el ornato, el medicinal, el comercio y las mascotas, llegando a la conclusión de que algunas culturas pueden aportar su conocimiento tradicional para darle así un uso racional a la fauna (Tejada et al. 2006; MonroyVilchis et al. 2008; Da Silva and Xavier 2010). Es tan importante esta relación del hombre con la fauna que, por ejemplo, se establecieron en la Argentina pautas de manejo sustentable de

Recibido: 29 de abril de 2016

Aceptado: 13 de febrero de 2017 
varias especies consideradas de valor especial, como el caso de proyectos de protección de hábitat para especies como el carpincho (Hydrochaeris hydrochaeris) y la nutria (coipo) (Myocastor coypus) (Bolkovic and Ramadori 2006; Bo et al. 2010).

Conocer el uso que se le da a la fauna silvestre cobra importancia si es que la situación de uso se presenta cerca o en lugares que se consideran protegidos, como es el caso de la Reserva Natural Otamendi (RNO), en el centroeste de Argentina. A través del tiempo, este espacio sufrió cambios que repercutieron en la sociedad que lo rodea. Para los pobladores de la zona ha sido comúnmente un lugar de esparcimiento, de pesca y de caza de animales para alimentación y comercialización. En la actualidad, la RNO forma parte del Sistema de Áreas Protegidas Nacionales de la República Argentina bajo la tutela de la Administración de Parques Nacionales. Tras la creación de la RNO en 1990, las actividades tradicionales de pesca y caza se convirtieron en usos ilegales según la legislación nacional, que prohíbe la caza y cualquier otro tipo de acción sobre la fauna en las Reservas Naturales. Si bien con los años estas actividades mermaron, siguen hasta el día de hoy, sobre todo como actividades de subsistencia por miembros de familias numerosas con necesidades básicas insatisfechas (Méndez 2012). En general, se asocia a la cacería (incluso la de subsistencia) como un factor importante en la desaparición de especies. La cacería de subsistencia, en los casos extremos puede hacer disminuir o desaparecer algunas especies con valor cinegético (Giraudo and Abramson 2000), por lo que es penada por la ley descrita.

Frente a esto, cabe resaltar la importancia de la inclusión de la comunidad y de sus conocimientos tradicionales dentro de la planificación del uso de fauna sostenible y de la conservación de áreas naturales (Pautasso 2003). Es aquí donde reside el valor de conocer dichos usos. Existen registros de uso de fauna en el área, en la región del Bajo Delta del Rio Paraná, donde Quintana et al. (1992) describieron el uso pasado y actual de la fauna silvestre, y la importancia de este recurso para el poblador local. Al ser una región con problemas socioeconómicos, la caza y la pesca fueron la base de ingreso de sus habitantes. La presión humana y las inundaciones que se presentaron en el momento de su estudio pusieron a algunas especies en estado delicado, lo cual hizo necesario la implementación de acciones de manejo para prevenir el deterioro de la fauna. En el barrio del Paraje de los Ciervos, colindante a la RNO, según los pobladores persiste la caza deportiva y de subsistencia en la zona por parte de la comunidad (García and Nale 2004). Un ejemplo de ello es el coipo, considerado un recurso por muchos pobladores locales de la Argentina debido al valor de su piel y, en menor medida, a su carne. Su uso puso en evidencia el conflicto que surgió en el área luego de la instauración de la reserva y la consecuente prohibición de continuar cazando a esta especie (Méndez 2012).

El estudio de los usos de la fauna silvestre permite dilucidar las percepciones sobre ésta, dado que las percepciones representan el resultado de aplicar nuestro conocimiento a situaciones particulares (Vodouhê et al. 2010). La importancia de un determinado animal para cierta sociedad depende de cómo se concibe y qué representa dentro de este grupo social. Su simbolismo y valor se vinculan con las percepciones, los conocimientos y los significados de cada individuo o de la comunidad en relación a esa especie (Wawrzyk 2013). Se han realizado estudios sobre percepciones de diferentes pobladores locales, por ejemplo, acerca de las reservas naturales (Jianying Xu et al. 2006; Hernández et al. 2008; Vodouhê et al. 2010), con una percepción, en general, de beneficio por parte de las comunidades aledañas, o un total desconocimiento del manejo de éstas y de la biodiversidad. Esto muestra una valoración utilitaria de la naturaleza y una preferencia por el uso de especies domésticas en la vida cotidiana (Campos et al. 2013; Wawrzyk 2013; Richeri et al. 2013; Trillo et al. 2016). Las percepciones pueden ser dilucidadas a través de las referencias al uso ya que gracias a nuestros patrones culturales estructuramos nuestros conocimientos y percepciones de forma individual y colectiva. Esto determinará los cursos de acción y las decisiones tomadas hacia la fauna (Navarro 2005). Identificar las percepciones acerca de la fauna puede producir información útil para la toma de decisiones de conservación y en la resolución de conflictos entre los pobladores locales y las autoridades (Vodouhê et al. 2010).

Nuestro objetivo general fue estudiar la relación entre los pobladores de dos barrios aledaños a la Reserva NaturalOtamendi(RNO) y la fauna silvestre. Los objetivos particulares fueron: a) identificar los principales usos que las comunidades aledañas a la RNO le dan a la 
fauna silvestre, $\mathrm{y}$ b) reconocer las percepciones de la fauna silvestre de vertebrados de la RNO por parte de las comunidades aledañas a la RNO. Se espera que los usos de la fauna silvestre no difieran a través del tiempo para la región, y que se pueda reconocer diferentes percepciones según las ocupaciones de los pobladores locales.

\section{MATERIALES Y MÉTODOS}

\section{Área de estudio}

La RNO se encuentra ubicada en el partido de Campana, a $65 \mathrm{~km}$ de la Ciudad de Buenos Aires. El área posee ambientes naturales característicos de las ecorregiones Pampeana y del Delta del Paraná, que están muy modificadas y poco representadas en los sistemas de áreas protegidas del país (Administración de Parques Nacionales 2004). Está cobijada bajo la Ley No 22.351 de Parques Nacionales, Monumentos Naturales y Reservas Nacionales y los Decretos $\mathrm{N}^{\circ}$

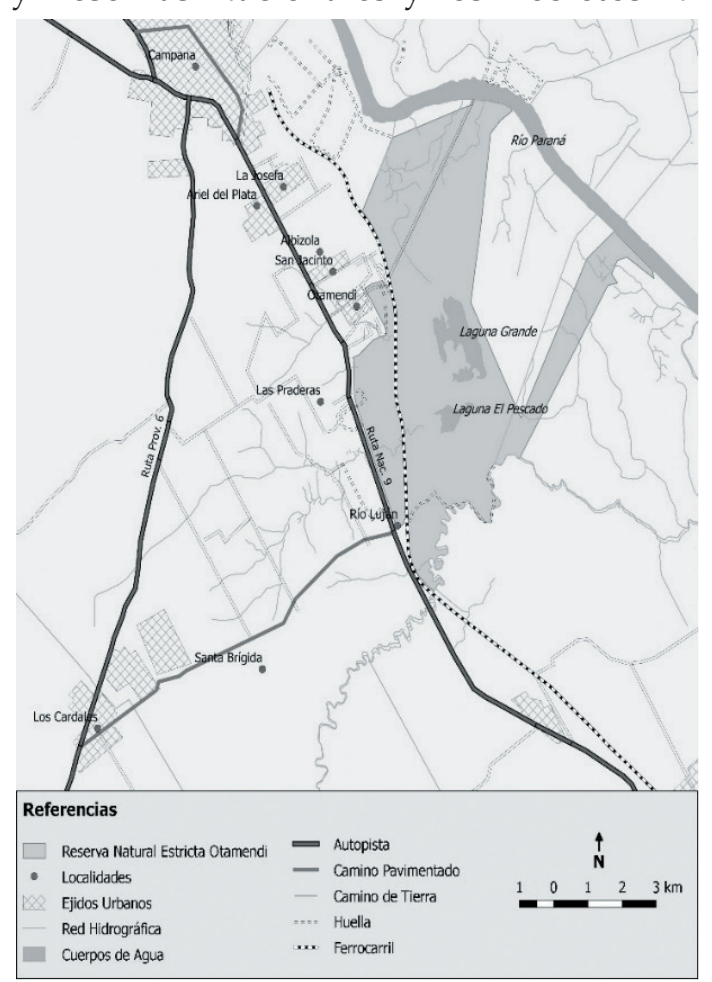

Figura 1. Mapa de ubicación de los sitios de estudio en la localidad de Campana, Provincia de Buenos Aires, Argentina. Fuente: elaborado por Juan Pablo Venturini, en base a información de Administración de Parques Nacionales e Instituto Geográfico Nacional.

Figure 1. Location map of the study sites in the Campana municipality, Buenos Aires province, Argentina. Source: Juan Pablo Venturini, based on information of National Parks Administration and National Geographic Institute.
2148/90 y 453/94 de creación del régimen de las Reservas Naturales Estrictas, Reservas Naturales Silvestres y Reservas Naturales Educativas (Administración de Parques Nacionales 2015).

En los alrededores de la RNO se sitúan los barrios Ingeniero Rómulo Otamendi y Estación Río Luján (Figura 1), caracterizados por una población con situaciones de pobreza, desempleo y distintas problemáticas sociales (Méndez 2012). Al barrio Ingeniero Rómulo Otamendi se lo considera "dormitorio", es decir, sus habitantes generalmente desarrollan sus actividades fuera de él, trabajando (o aspirando a trabajar) en alguna de sus industrias. Hay diversos comercios, casas de comidas para llevar y algunos vecinos crían animales para autoconsumo; al año 2008 contaba con $~ 5000$ habitantes (Méndez

Tabla 1. Entrevista realizada a los pobladores locales acerca del uso de fauna, se tuvieron en cuenta tres ejes principales: datos acerca del poblador, usos de fauna e historia de uso.

Table 1. Interview done to the local people about wildlife use, we used three principal axes: people data, wildlife use and history of use.

\begin{tabular}{|c|c|c|}
\hline $\begin{array}{l}\text { ENTREVISTA A } \\
\end{array}$ & POBLADOR & \\
\hline Entrevista No. & & echa: \\
\hline DATOS SOBRE EL IN & ORMANTE & \\
\hline 1. Barrio & & \\
\hline 2. Nombre del entrevistado: & Sexo: F M & Edad: \\
\hline 3. Tiempo de residencia: & & \\
\hline 4. Lugar de procedencia: & & \\
\hline 5. Ocupación: & & \\
\hline
\end{tabular}

REFERENCIAS ACERCA DE LA FAUNA (ENFASIS EN EL USO)

6. ¿Usa animales silvestres? Sí No

7. Si la respuesta anterior es positiva, ¿cuáles? ¿Sabe que animales se silvestres se usan en la zona? Sí No

8. Si la respuesta anterior es positiva, ¿cuáles? 9. ¿Para qué se usa?

$$
\begin{array}{lll}
\text { Alimento } & \text { adorno medicinal comercio } \\
\text { mascota } & \text { valor deportivo Otros }
\end{array}
$$

10. ¿Qué parte del animal es utilizada? ¿Por qué?

11. ¿Con qué frecuencia lo hace? Otros:

12. ¿En quéépoca del año? Semana Mensual

12. ¿En querano Otoño Invierno Primavera

13. ¿Hay más o menos que antes? ¿Por qué?

HISTORIA DE USO

14. ¿Hace cuánto se usan estos animales?

15. ¿Cómo aprendió esta práctica de uso de fauna?

16. ¿Piensa que la transmite o transmitió esta práctica de uso de fauna a otras personas?

Sí No No ¿De qué manera?
17. ¿Siente algún impedimento para continuar con esta práctica de uso? En qué se vio favorecido o perjudicado?

18. ¿Tiene usted o su familia una dependencia directa de estos

19. ¿En qué lugares solia o suele obtener los animales para su

20. Otra información (detalles de comercio, artes de caza, etc.) 
2012). El barrio Estación Río Luján cuenta con 200 habitantes. Al año 2011, muchas familias tenían niños en edad escolar, los empleos de los adultos eran precarios, las casas no contaban con cloacas ni gas natural y no posee establecimientos educativos (Méndez 2012).

Entre septiembre de 2014 y abril de 2015 se realizaron entrevistas semi-estructuradas a miembros de las comunidades mencionadas (Tabla 1). Las entrevistas permitieron obtener información acerca de las referencias de usos de fauna (ya que las referencias son lo que los pobladores dicen que hacen, lo que es diferente de que el investigador participe y documente actividades de uso de fauna), y dilucidar las percepciones (Lucca Irizarry and Berríos Rivera 2009). Para obtener una muestra de la población se utilizó la técnica en investigación cualitativa "bola de nieve", que consiste en que cada informante recomiende al investigador una o más personas de su círculo de conocidos (Quintana et al. 1992; Guber 2004). Este proceso es repetitivo: los informantes refieren al investigador a otros informantes quienes son contactados y después ellos refieren a otras personas, y así sucesivamente (Noy 2014).

Se contactaron pobladores a través de profesionales de distintos programas de la Administración de Parques Nacionales y de la Dirección Nacional de Conservación de Áreas Protegidas, esperando que pudieran referir a algunos pobladores más para entrevistar. El reconocimiento de actores sociales, como maestras, estudiantes, miembros dela sociedad de fomento, etc., también fue clave para poder establecer un nexo con la comunidad. A cada entrevistado se le explicó con antelación que las entrevistas se iban a realizar en el marco de una tesis de maestría en ciencias ambientales de la Universidad de Buenos Aires, que iba a tratar sobre el uso de fauna silvestre, se le pidió su consentimiento y se le dio la opción de responder de forma anónima. Todas las entrevistas se realizaron de forma presencial intentando establecer una conversación fluida con los entrevistados.

En la entrevista semiestructurada, el entrevistador crea con anticipación las preguntas, pero posee la libertad de alterar el orden de las mismas, sin olvidar los objetivos de la investigación. Este tipo de entrevista busca construir un cuadro acerca de uno o varios puntos de interés (Lucca Irizarry and Berríos Rivera 2009). Permite documentar el conocimiento ecológico tradicional, dirigir las discusiones en la medida necesaria para cubrir temas específicos más a fondo, discutir y describir puntos de vista de los temas, y establecer conexiones basadas en dichos puntos de vista más allá de las preguntas redactadas de antemano por el investigador (Abba and Casini 2010).

La entrevista realizada pretendió obtener información de las referencias al uso de la fauna silvestre, y es una modificación de la entrevista aplicada por Quintana y colaboradores (1992). Para asignar un uso cualitativo a las especies mencionadas se consideraron categorías establecidas de uso (i.e., alimento, adorno, medicinal, comercio, mascota), qué partes se usan y con qué frecuencia, y en qué época del año (MonroyVilchis et al. 2008; Tejada et al. 2006). Se relevaron, además, datos sociodemográficos como edad, tiempo de residencia y ocupación (Tabla 1). Las percepciones acerca de la fauna silvestre emergieron a partir de los resultados de las entrevistas. La información se categorizó teniendo en cuenta los testimonios de los entrevistados; los testimonios resaltaron los tres temas más referidos en la mayoría de entrevistas: la abundancia de fauna, la prohibición de cazar y el mascotismo.

\section{Resultados \\ Actores sociales}

Se entrevistaron 44 personas, clasificadas en dos grupos de actores sociales: pobladores locales mayores de edad $(n=18)$ y estudiantes de nivel secundario $(n=26)$. En el primer grupo se obtuvo información de 12 mujeres y 6 hombres, cuyas edades oscilaron entre 23 y 64 años, con un promedio de 45 años. El 50\% de las personas del primer grupo ha residido por más de 27 años en los barrios Otamendi y Estación Río Luján o son pobladores de la zona de Islas Río Paraná de las Palmas en la sección del Partido de Campana (Figura 1, Tabla 2). El segundo grupo constituye parte de la población de adolescentes, con un promedio de edad de 14 años, donde el 65\% pertenece al sexo femenino.

\section{Especies usadas por las comunidades}

El $80 \%$ de los entrevistados afirmó no usar a la fauna silvestre y el $20 \%$ menciona el uso de especies como el carpincho (Hydrochoerus hydrochaeris), el coipo (Myocastor coypus), el zorro gris (Lycalopex gymnocercus), el ciervo (Blastocerus dichotomus), el ñandú (Rhea americana), el lagarto overo (Salvator 
Tabla 2. Ocupaciones de los entrevistados.

Table 2. Occupations of respondents.

\begin{tabular}{|c|c|}
\hline $\begin{array}{l}\mathrm{N}^{\circ} \text { de } \\
\text { entrevistado }\end{array}$ & Ocupación \\
\hline 1 & $\begin{array}{l}\text { Empleado administrativo (Dirección de } \\
\text { Turismo del Barrio Otamendi) }\end{array}$ \\
\hline 2 & Empleado en industria (SIDERCA) \\
\hline 3 & Turismo \\
\hline 4 & Preceptor de escuela \\
\hline 5 & Portera de escuela \\
\hline 6 & Embarcador \\
\hline 7 & $\begin{array}{l}\text { Presidente de Sociedad de Fomento } \\
\text { (Barrio Otamendi). }\end{array}$ \\
\hline 8 & Cazador \\
\hline
\end{tabular}

merianae) y diversos peces del río Paraná. El $40 \%$ de los entrevistados que no usan fauna afirma saber qué animales se usan en la zona. El carpincho fue el más mencionado por los pobladores, seguido por el coipo y las aves. Algunos animales fueron mencionados a nivel de especie, mientras que las aves y los peces se mencionaron como grupo (Figura 2).

\section{Tipos de uso de la fauna}

Los entrevistados refirieron tres categorías de uso más frecuente: alimento, comercio y mascotismo. Los usos como adorno o deporte se mencionaron poco. La categoría de uso medicinal no se refirió y el $26 \%$ de los entrevistados no citó ningún uso (Figura 3). Cuando la fauna es utilizada como alimento, los pobladores afirmaron que es para consumo propio y que depende de la necesidad económica del momento. Cuando la fauna no se consume como alimento, se vende la piel y la carne. Por ejemplo, al año 2015, para un coipo, un poblador estima un valor de $\$ 100$

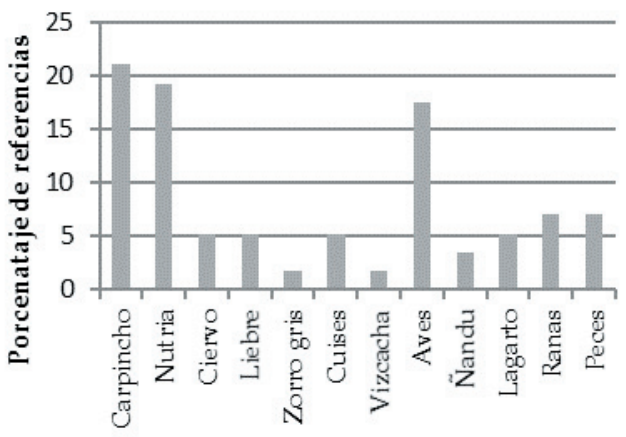

Taxa mencionados

Figura 2. Fauna que se sabe que se usa en la zona referida por los entrevistados. NS/NR: No sabe/No responde.

Figure 2. Wildlife that is known that is used in the zone referred by interviewees. NS/NR: Do not know/Do not respond.

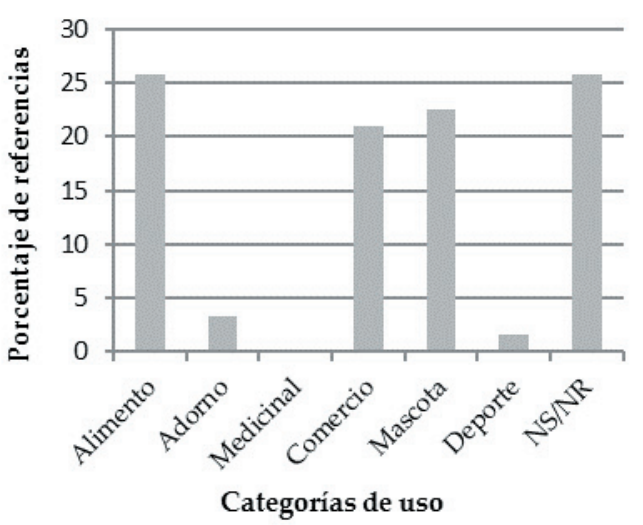

Figura 3. Categorías de uso de fauna referidas por los entrevistados NS/NR: No sabe/No responde.

Figure 3. Wildlife use categories referred by interviewees. NS/NR: Do not know/Do not respond.

AR: el cuero \$30 AR y la carne \$70 AR. Si bien el volumen de caza no fue estimado, en un hogar se observaron acondicionados 11 cueros de coipo y 20 de carpinchos.

Los animales usados como mascotas son principalmente aves, como los loros y las aves canoras (por ejemplo, el corbatita común, Sporophila caerulescens) que se atrapan con este fin. No obstante, un poblador de las islas (Río Paraná), antiguo cazador de la zona, afirma tener dos carpinchos como mascota. Los pobladores mencionaron el uso para caza deportiva del ciervo de los pantanos, cuyas cabezas son consideradas como trofeo y tienen valor en el mercado a pesar de que su caza es ilegal.

La frecuencia de uso de fauna más referida fue la diaria $(11 \%)$ cuando es un medio de subsistencia. La caza semanal $(7 \%)$ y la mensual (5\%) fueron referidas menos veces, y la caza anual no se mencionó. El 77\% de los entrevistados no respondió a la pregunta. La estación del año que más se menciona para la caza es el invierno (18\%), porque los animales no están en época reproductiva y son menos agresivos (como el ciervo). Algunas personas afirman que en verano se caza mal, sin embargo, es otra época referida para esta actividad (14\%). Otros mencionan que la caza se realiza todo el año (9\%), o en primavera $(9 \%)$ y muy pocas veces se menciona el otoño (2\%). El $48 \%$ de los entrevistados no respondió esta pregunta.

\section{Breve historia de uso}

Para establecer una historia de uso de fauna en la zona no se tuvo en cuenta el grupo de estudiantes de educación secundaria ya que 
el 77\% afirma no conocer la fauna que se usa. Del total de pobladores locales mayores de edad, 10 aportaron respuestas. Según ellos, los animales se han usado desde hace mucho tiempo o siempre. La práctica de la caza era una actividad que se transmitía de padres a hijos, y también se practicaba en los espacios de socialización con amigos.

Los lugares mencionados en donde se solía (o se suele) cazar animales para su uso incluyen sectores del área actual de la RNO, los canales cercanos al Río Paraná, lagunas, arroyos y bañados. Esta práctica se ha venido dejando de lado, aunque algunos pobladores (22\%) consideran que la transmitió de alguna manera, yendo a cazar con los hijos o con amigos. Una razón de que la práctica de la caza sea menos frecuente es que ya no se puede practicar donde hoy es la reserva. Con el papel de la reserva como ente protector de la naturaleza, con el desinterés de la población adolescente y con el hecho de que dejó de ser un medio de subsistencia, "se dejó de cazar para consumo, ya hay trabajo; entonces no se caza".

\section{Percepciones detectadas en los entrevistados acerca de la fauna silvestre de vertebrados.}

Del análisis de las entrevistas realizadas y los testimonios emergentes (detallados entre comillas) se pudo inferir que las percepciones de los actores sociales acerca de si hay más o menos fauna que antes son, al menos en parte, contradictorias. Además, la percepción depende del entorno en que se desempeña cada entrevistado. Se evidenciaron cinco percepciones

"Hay menos fauna que antes". Al preguntarle a las personas si hay menos o más animales que antes, la mayoría (59\%) percibe que hay menos que antes. Los pobladores expresan que esto se debe a que la gente los caza (furtivismo), a la pérdida de hábitat y crecimiento de la población humana, que produce aumento de las urbanizaciones y contaminación.

"Hay más fauna que antes". Sólo tres entrevistados perciben que hay más fauna que antes ya que hay más cuidado gracias a la reserva. Desde la perspectiva de un cazador, hay más fauna ya que muchas personas dejaron de cazar. En este sentido, este actor social se ve favorecido pues puede cazar más en donde haya oportunidad.

"La caza no está permitida en la reserva". Que la caza sea la principal razón referida por la cual disminuyó la fauna, y que el
80\% entrevistado afirmó no usar animales silvestres, sugiere que la mayoría de la gente percibe a esta práctica como algo prohibido y con efectos negativos sobre la fauna.

"La caza como medio de subsistencia". Al ser la caza una práctica incorporada en la vida diaria, el uso de fauna es frecuente y depende en cierta parte de las oportunidades para conseguir animales, como las subidas de los ríos. En este caso, que exista una reserva se convirtió en una oportunidad para alguien que depende de esta práctica para vivir y reside cerca.

"No hay diferencia entre animal doméstico y animal silvestre". Al preguntarles a los estudiantes de secundario acerca del uso de animales silvestres, algunos nombran animales como el gato o el perro, y su uso se refiere a la mascota. Estas características desdibujan la distinción entre doméstico y silvestre.

\section{DISCUSIÓN}

\section{Especies usadas por las comunidades}

Los animales que más se usan en la zona aledaña a la RNO incluyen a los mamíferos, como el carpincho y el coipo, dos de las especies más representativas del Delta del Paraná y principal recurso de fauna silvestre para muchos de sus pobladores (Bo et al. 2010). También las aves son mencionadas con frecuencia como fauna utilizada en la zona. Ambos resultados concuerdan con estudios previos en la región (Quintana et al. 1992), mientras que del resto de fauna mencionada por estos autores hay un bajo porcentaje de uso en el grupo de los anfibios y reptiles.

Las condiciones del entorno (e.g., las condiciones atmosféricas, la estacionalidad, el estado del lugar y su forma de acceso) determinan la fauna que se usa; esto se aplica al humedal, que hace parte y rodea la reserva (e.g., se caza al coipo con trampa de cepo sólo en ambientes topográficamente bajos y permanentemente inundados [Bo et al. 2010]). Cuando hay sudestadas o lluvias copiosas, en los humedales aumenta el nivel del agua y algunos animales se ven obligados a transitar sobre este nivel. Es así que existe mayor fauna disponible y, por lo tanto, resulta una época propicia para cazar. Al considerar este tipo de caza oportunista (por ejemplo, en los pulsos de inundación de los humedales) podría disminuir la presión de caza sobre especies de roedores (coipo y carpincho) y 
aumentar sobre otras (e.g., el lagarto overo). Esto podría dilucidarse con un estudio más profundo en relación a las variables climáticas y las oportunidades de caza.

Aunque existen similitudes entre estudios acerca de la fauna que se usa, en el tiempo, el elenco de fauna empleada es variable. Por ejemplo, la rana criolla Leptodactylus latrans, una especie común en la zona, fue un recurso tradicional e importante en todo el Delta hasta su drástica disminución luego de la inundación de 1982-1983 (Quintana et al. 1992). En la actualidad sólo se la caza en baja proporción para consumo local, lo que concuerda con los testimonios actuales de algunos pobladores. Otro caso es el del lagarto overo, que para la época en la que se llevó a cabo el estudio de Quintana et al. (1992), su caza estaba aumentando debido a la exportación de cueros. Estos animales se siguen cazando (pocos, según un poblador) y se los entrega vivos a quienes los requieren. Sin embargo, no fue claro el uso posterior a la entrega. El coipo fue cazado de manera intensa en todo el país, razón por la que pasó por diferentes etapas tendiendo a un uso sustentable: desde permitir capturas sin cupo (hasta 1998), hasta proponer una temporada oficial de caza extendida desde mediados de otoño hasta mediados de primavera, ya que en esa época la piel es más espesa y tiene mayor valor comercial (Bo et al. 2010).

\section{Tipos de uso de la fauna}

A las especies de fauna capturadas se les da diferentes usos. Esto puede diferir de un poblador a otro, independientemente de las especies que se mencionaron antes ya que las situaciones individuales de cada poblador (e.g., acceso a recursos, sueldo fijo, tenencia de animales, etc.) son variables y pueden cambiar en el tiempo. La predominancia de los usos como alimento y caza comercial relevada coincide con lo registrado por Quintana et al. (1992). La fauna silvestre ha sido utilizada desde antaño con diversos propósitos, que incluyen la alimentación humana. Sin embargo, en la actualidad, sólo algunos pobladores de muy bajos recursos, como se observó en la comunidad aledaña a la reserva, se deben valer de cualquier fuente de alimento para subsistir. En estos casos la caza de animales silvestres juega un rol fundamental para la obtención de proteína animal fresca (Pautasso 2003). Esto podría explicar por qué algunos pobladores locales los usan como alimento. Como su subsistencia depende de todos los aportes posibles, también se aprovecha para comerciar animales vivos o partes como el cuero.

En este estudio encontramos casos de tenencia de fauna silvestre como mascota. Ya Quintana et al. (1992) señalaron que es frecuente la captura viva de varias especies para ser vendidas como mascotas. Los animales son cazados o capturados, muchas veces crías de adultos y son terminados de criar en las casas; se crea una importante relación con estas especies en particular puesto que se convierten en un miembro más de la familia. En cuanto al uso deportivo, se menciona al ciervo de los pantanos (aunque el número de referencias a este uso fue bajo). La especie se considera de valor especial pues está declarada monumento natural según el decreto 12209/98. En general, hoy en día los ciervos son cazados por personas ajenas a las comunidades estudiadas. Aunque hay cazadores que son antiguos habitantes de la zona, también se reconocen como posibles cazadores a la gente que va a pasar el fin de semana a las quintas o a pescar, como también a los nuevos pobladores (ocupantes), que tienen una cultura diferente (Méndez 2012).

El uso de los recursos naturales en un área determinada es el resultado de factores históricos, sociales y económicos. Se hace evidente que el impacto de la cacería sobre la fauna silvestre no es estrictamente biológico si no también sociocultural, por lo que las alternativas para la utilización de recursos y su manejo sustentable deberían ser viables desde ambos puntos de vista (Giraudo and Abramson 1998). De esta manera, conocer los usos de la fauna permite resaltar su importancia como recurso natural para las poblaciones que viven de su uso, su impacto, y permite considerar tópicos novedosos para el manejo y la conservación que conduzcan a un uso sustentable (Pautasso 2003).

\section{Percepciones detectadas en los entrevistados acerca de la fauna silvestre de vertebrados}

Según Méndez (2012), las tierras que hoy constituyen la RNO han sido históricamente un lugar de esparcimiento, de pesca y de caza de coipos para alimentación y comercialización de pieles de los vecinos de los barrios Otamendi, Río Luján y de la zona en general. Con la creación de la reserva, estas actividades tradicionales, salvo el esparcimiento, se convirtieron en ilegales. La caza no está permitida dentro de las áreas protegidas, y si bien con los años ha mermado, 
se considera que sigue actualmente en la reserva. Sin embargo, frente a esta percepción se plantea una situación problemática ya que algunas personas practican la caza como medio de subsistencia. Por esta razón se crea un conflicto entre la subsistencia de algunos pobladores y la conservación de la fauna en la RNO. Según Méndez (2012), en el barrio Río Luján algunos vecinos recuerdan que en la época de la crisis del 2001 se cazaban coipos y cuises (Cavia aperea) dentro de la RNO y luego pasaban por la calle vendiendo la carne. Otro vecino comentó que antes de la creación de la RNO entraba para cazar patos (posiblemente Dendrocygna y Anas) porque había muchísima cantidad y porque los destinaba a alimento de la familia.

La protección que ejerce la reserva motiva a las personas a dejar de utilizar el recurso fauna y a recurrir a otras formas de subsistencia. De esta manera, la cacería es penada y reprimida no sólo por los organismos gubernamentales sino también por los no gubernamentales y por la sociedad en general (quizá sin tener en cuenta el verdadero impacto sobre las especies y los ecosistemas, ni las necesidades sociales y culturales que la motivan) (Pautasso 2003). De esta manera se pierden prácticas tradicionales que además de enriquecer la cultura pueden ser tomadas en cuenta para darle un uso equilibrado a los recursos.

Méndez (2012) afirma que los vecinos reconocen que falta educación ambiental y que se desconoce la situación de las especies en los barrios cercanos a la RNO. A esto se suma el hecho de no llegar a acuerdos con los pobladores y de no tener en cuenta sus situaciones socioeconómicas desde que se instauró la reserva, chocando con las tradiciones de algunos para los cuales la caza es importante. Por eso, la conservación no puede limitarse al establecimiento de áreas protegidas o a la creación de regulaciones legales para el uso de los recursos naturales. Se necesita contar con el apoyo de las comunidades humanas, considerando su diversidad de percepciones, apreciaciones, valoraciones y actitudes que ellas tienen hacia la biodiversidad (Campos 2013). Al año 2015, el Plan de Gestión de la RNO plantea que es necesario generar espacios de intercambio y debate junto a los pobladores locales y profundizar temas como el uso tradicional de fauna silvestre, dado que el patrimonio inmaterial es representativo y basado en el sentido de comunidad (Administración de Parques Nacionales 2015). Ello podría considerarse un paso para llegar a generar usos sustentables por parte de la figura que representa autoridad, algo que debería ponerse en acción y evaluar con frecuencia, ya que además mejoraría la relación de la comunidad con la reserva.

Los resultados obtenidos permiten plantear, por primera vez para este sitio de estudio, que los cambios en la sociedad semi-rural que rodea la reserva influirían en las percepciones de las personas. Que los pobladores perciban que hay más o menos fauna silvestre que antes y que las razones sean por causa humana, sugiere el reconocimiento del impacto antrópico sobre ésta. El papel de la reserva ha sido importante a través del tiempo ya que gracias a ella hay más cuidado de los animales (según los pobladores), pero no queda claro qué sucede fuera de ésta. Al dilucidar estas percepciones se evidencian dos tipos de relaciones que los pobladores mantienen con la fauna: una relación distante para unos, algo que quizá se ve, pero no se conoce, o una relación cercana y de beneficio propio para otros.

\section{Valor de uso de fauna autóctona silvestre como recurso}

Para los pobladores que practican la cacería, los mamíferos son los animales más importantes y los que así representan un valor de uso. Se destaca el coipo, para el que la principal técnica de captura descrita por los entrevistados es la trampa por cepo, que no tendría un efecto selectivo importante sobre la proporción de sexos. Por ello resulta una técnica sustentable que permite mantener el recurso en el tiempo (Bo et al. 2010; Courtalon et al. 2015). La caza de coipos es una actividad habitual, pero al 2004, pobladores de la región la desaprobaban por considerarla innecesaria y peligrosa (García and Nale 2004). Esto evidencia que, en casi 10 años, algunas modalidades y usos que se le da a la fauna no han cambiado. Desde el 2001 el Proyecto Nutria intenta implementar un plan de acción con medidas concretas que ayuden de manera efectiva al manejo sustentable de la especie y de los humedales que habita, contribuyendo así a su conservación y al mejoramiento de la calidad de vida de los pobladores locales que la utilizan (Bó et al. 2006; Bo et al. 2010).

El carpincho es una especie muy utilizada en toda su área de distribución. Es uno de los mamíferos más llamativos de los humedales argentinos, además de ser escasamente estudiado en el medio silvestre en el país 
(Bolkovic and Ramadori 2006). Aunque, según los entrevistados en el presente estudio, del carpincho se aprovecha todo, en la actualidad el principal subproducto utilizado es la piel. La caza de esta especie está prohibida para la provincia de Buenos Aires, pero en el área de estudio se lleva a cabo, por lo que aún se deben sentar las bases para su uso sustentable. Se deben rescatar las técnicas de caza tradicionales como el uso de la cimbra, y tener en cuenta las densidades poblaciones actuales.

El ciervo de los pantanos fue objeto de acciones directas de protección, como la creación de unidades de conservación en áreas naturales donde se encuentra la especie, como es el caso de la RNO (D'Alessio et al. 2012). Posee el máximo nivel de protección legal. Además, a nivel nacional está prohibida la caza, captura y comercialización de esta especie, y está declarada en peligro de extinción ( $\mathrm{D}^{\prime}$ Alessio et al. 2012) e incluida en el libro rojo de mamíferos amenazados de Argentina (Ojeda et al. 2012). Pese a esta reglamentación, la caza de ciervo de los pantanos en la región ha sido practicada por la población isleña local, y en general no puede considerarse una actividad de subsistencia. Si bien se lo consume, el origen de su caza ha estado asociado a costumbres culturales muy arraigadas. Hoy en día, su caza alcanza niveles alarmantes en algunos sectores, y esta es una de las causas fundamentales que explicarían numerosas extinciones locales en gran parte de las islas del Paraná. Así, existe un desconocimiento de su estado de conservación actual y de la legislación que lo cubre por parte de los pobladores locales (D'Alessio et al. 2012).

Es evidente que la práctica de la caza en la zona aledaña a la RNO es una actividad vigente, sin embargo, su estigmatización choca con las tradiciones de algunos pobladores. Por esta razón, las estrategias de manejo de recursos naturales deben ser planificadas e implementadas de forma conjunta a partir de las propuestas de manejo tradicional de los pobladores locales y políticas provinciales (Giraudo and Abramson 2000), puesto que las áreas protegidas por si solas no son suficientes para garantizar la conservación de la biodiversidad (García and Nale 2004).

\section{Conclusiones}

Conocer los usos de la fauna permite establecer su importancia como recurso natural para las poblaciones que viven de su uso y considerar tópicos novedosos para el manejo y la conservación. Por esta razón se deben ampliar y actualizar los estudios en este tema en la zona de estudio. Los mamíferos son los animales más importantes para los pobladores que cazan fauna silvestre en la zona aledaña a la RNO. Es necesario resaltar el valor de uso de las especies más usadas en el área de estudio, como el coipo o el carpincho, junto con los pobladores, indistintamente de su ocupación, para establecer pautas sustentables de uso locales que contemplen el efecto de la cacería de subsistencia y las prácticas tradicionales de la comunidad. La información que pueden brindar los cazadores locales acerca de la fauna silvestre debe considerarse una forma de conocimiento para tener en cuenta en los programas de conservación y manejo. Esto se puede lograr aprovechando los espacios comunes de reunión como la escuela o las juntas de acción comunal, con el objetivo de acercar a la comunidad al conocimiento de su entorno y de esta manera poder apreciarlo y aprovecharlo sustentablemente.

Agradecimientos. A los pobladores locales, padres, madres de familia, amas de casa, docentes, que se tomaron el tiempo de responder la entrevista. A M. Méndez, M. Romiti y L. Staropoli por permitir acercarnos a la comunidad aledaña a la reserva haciendo el contacto con ellos.

\section{REFERENCIAS}

Abba, M., and M. Cassini. 2010. A comparison of two methods for acquiring ecological data on armadillos from Argentinean pampas: field work vs interviews. Interciencia 35:450-454.

Administración de Parques Nacionales. 2004. Plan de manejo de la Reserva Natural Otamendi, 2005-2009.

Administración de Parques Nacionales. 2015. Plan de gestión de la Reserva Natural Otamendi, 2016-2021.

Bó, R. F., G. Porini, M. J. Corriale, and S. M. Arias. 2006. Proyecto Nutria. Estudios ecológicos básicos para el manejo sustentable de Myocastor coypus en Argentina Pp. 93-104 en M. L. Bolkovic and D. Ramadori (eds.). Manejo de Fauna Silvestre en Argentina. Programas de uso sustentable, editado. Dirección de Fauna Silvestre, Secretaría de Ambiente y Desarrollo Sustentable, Ministerio de Salud y Ambiente de la Nación, Buenos Aires, Argentina.

Bó, R., P. Courtalon, R. Fernández, and G. Porini. 2010. El manejo sostenible del Coipo (Myocastor coypus) en el Delta del Paraná y otros humedales de Argentina. Diez años del proyecto "Nutria" Pp. 119-127 en Delta del Paraná: Historia, presente y futuro. Trabajos completos Simposio Científico Académico Delta del Paraná, 4 y 5 de octubre, San Fernando, Argentina. 
Bolkovic, M. L., and D. Ramadori (eds.). 2006. Manejo de Fauna Silvestre en la Argentina. Programas de uso sustentable. Dirección de Fauna Silvestre, Secretaría de Ambiente y Desarrollo Sustentable, Buenos Aires, Argentina.

Campos, C. M. 2013. Percepción de la biodiversidad. Ecología Austral 23:145-146.

Campos, C. M., J. Nates, and P. Lindemann-Matthies. 2013. Percepción y conocimiento de la biodiversidad por estudiantes urbanos y rurales de las tierras áridas del centro-oeste de Argentina. Ecología Austral 23:174-183.

Courtalon, P., R. Bó, F. Spina, N. Jiménez, L. Cantil, R. Fernández, and G. Porini. 2015. Reproductive ecology of coypu (Myocastor coypus) in the Middle Delta of the Paraná River Argentina. Brazilian Journal of Biology 75:30-38.

D'Alessio, S., G. Aprile, B. Lartigau, P. Herrera, D. Cano, A. Eberhardt, L. Antoniazzi, I. Jiménez Pérez, A. Di Giacomo, N. Meyer, N. Fracassi, H. Cardozo, D. Varela, A. Parera, H. Ball, M. B. Aued, C. Figuerero, A. Fernández, Y. Lezcano, P. Cowper Coles, M. Sosa, N. Cowper Coles, G. Ramírez, and A. Kees. 2012. Ciervo de los Pantanos Blastocerus dichotomus (Illiger 1815). Pp. 249-280 en Manejo de fauna silvestre en la Argentina. Programa de Conservación de Especies Amenazadas. SAyDS y FHN Félix de Azara. Buenos Aires, Argentina.

Da Silva, T. S., and E. M. Xavier. 2010. Perception and use of fauna resources in communities surrounding a conservation unit in northeast Brazil. Acta Scientiarum. Biological Sciences 32:365-371.

García, M., and M. Nale. 2004. Informe sobre diagnóstico socio-productivo realizado a comunidades vinculadas a la Reserva Natural Otamendi: El Paraje Los Ciervos. Administración de Parques Nacionales. Dirección Nacional de Conservación de Áreas Protegidas. Dirección de Conservación y Manejo. Programa Asentamientos Humanos. Argentina.

Giraudo, A. R., and R. Abramson. 1998. Usos de la fauna silvestre por los pobladores rurales en la selva paranaense de Misiones. Tipos de uso, influencia de la fragmentación y posibilidades de manejo sustentable. Boletín Técnico de la Fundación Vida Silvestre, No. 42, Buenos Aires, Argentina. Pp. 48.

Giraudo, A. R., and R. Abramson. 2000. Diversidad cultural y usos de la fauna silvestre por los pobladores de la selva misionera: ¿Una alternativa de conservación? Pp. 233-243 en C. Bertonatti and J. Corcuera (eds.). La situación ambiental Argentina. Fundación Vida Silvestre, Buenos Aires, Argentina.

Guber, R. 2004. El salvaje metropolitano. Reconstrucción del conocimiento social en el trabajo de campo. Editorial Paidós, Buenos Aires, Argentina.

Hernández, H., L. Beltrán, H. Villarreal, and A. Ortega. 2008. Perceptions of a fishing community about benefits, environmental impacts and use of resources of Isla Cerralvo, a protected island in the Gulf of California, Mexico. Interciencia 33:604-609.

Jianying Xu, C. L., and B. J. Yihe Lu. 2006. Local people's perceptions as decision support for protected area management in Wolong Biosphere Reserve, China. Journal of Environmental Management 78:362-372.

Lucca Irizarry, N., and R. Berríos Rivera. 2009. Investigación cualitativa: Fundamentos, diseños y estrategias. Ediciones SM, Cataño, Puerto Rico.

Méndez, M. 2012. Propuesta de delimitación para una Zona de Amortiguamiento de la Reserva Natural Otamendi. Tesis de Licenciatura en Información Ambiental. Universidad Nacional de Lujan. Luján, Buenos Aires, Argentina. Pp. 342.

Monroy-Vilchis, O., L. Cabrera, P. Suárez, M. M. Zarco-González, C. Rodríguez-Soto, and V. Urios. 2008. Uso tradicional de vertebrados silvestres en la Sierra Nanchititla, México. Interciencia 33:308-313.

Navarro, M. 2005. Conocimientos y percepciones sobre la fauna por los habitantes de la Isla Cozumel. Tesis profesional para obtener el título de licenciado en biología. Universidad de Guadalajara. Centro Universitario de Ciencias Biológicas y Agropecuarias. Las agujas, Zapopan, Jalisco, México. Pp. 85.

Noy, C. 2008. Sampling Knowledge: The Hermeneutics of Snowball Sampling in Qualitative Research. International Journal of Social Research Methodology 11:327-344.

Ojeda, R., V. Chillo, and G. Díaz (eds.). 2012. Libro Rojo de Mamíferos Amenazados de la Argentina. Sociedad Argentina para el Estudio de los Mamíferos, Argentina.

Pautasso, A. 2003. Aprovechamiento de la fauna silvestre por pobladores rurales en la fracción norte de los bajos submeridionales de la provincia de Santa Fe, Argentina. Com. Mus. Prov. Cs. Naturales "Florentino Ameghino" (nueva serie) Santa Fe, Argentina 8:1-62.

Quintana, R., R. Bó, J. Merler, P. Minotti, and A. Málvarez. 1992. Situación y uso de la fauna silvestre en la región del Bajo Delta del Río Paraná, Argentina. IHERINGIA, Série.Zoología 73:13-33.

Racero-Casarrubia, J., C. Vidal, O. Ruiz, and J. Ballesteros. 2008. Percepción y patrones de uso de la fauna silvestre por las comunidades indígenas Embera-Katíos en la cuenca del río San Jorge zona amortiguadora del PNN-Paramillo. Revista de Estudios Sociales 31:118-131.

Richeri, M., M. B. Cardoso, and A. H. Ladio. 2013. Soluciones locales y flexibilidad en el conocimiento ecológico tradicional frente a procesos de cambio ambiental: estudios de caso en Patagonia. Ecología Austral 23:184-193.

Tejada, R., E. Chao, H. Gómez, R. E. L. Painter, and R. B. Wallace. 2006. Evaluación sobre el uso de la fauna silvestre en la Tierra Comunitaria de Origen Tacana, Bolivia. Ecología en Bolivia 41:138-148.

Trillo, C., B. Arias, and S. Colantonio. 2016. Uso y percepción del bosque por pobladores de diferente tradición cultural de la Laguna de Mar Chiquita, Córdoba, Argentina. Ecología Austral 26:007-016.

Vodouhê, F., O. Coulibaly, A. Adégbidi, and B. Sinsin. 2010. Community perception of biodiversity conservation within protected areas in Benin. Forest Policy and Economics 12: 505-512.

Wawrzyk, A. C. 2013. Saberes etozoológicos de los pastores andinos: su importancia para la conservación y manejo de la vicuña (Vicugna vicugna). Ecología Austral 23:156-164. 